\title{
Semi-Automated Cryo-EM Sample Loader for TEM SPA Democratization
}

Vojtěch Doležal $^{1}$, Václav Prajzner ${ }^{1}$, Martin Čechmánek ${ }^{1}$, Zuzana Hlavenková ${ }^{1}$, Ondřej Sháněl ${ }^{1}$, Stanislav Tománek $^{1}$, Markéta Červinková ${ }^{2}$, Daniel Němeček ${ }^{3}$ and Lingbo $\mathrm{Yu}^{1}$

${ }^{1}$ Thermo Fisher Scientific, United States, ${ }^{2}$ Thermo Fisher Scientific, Brno-Cernovice, Czech Republic, ${ }^{3}$ Thermo Fisher Scientific, Czech Republic

Broader adoption of the cryo electron microscopy (cryo-EM) Single particle analysis (SPA) workflow requires a reliable, easy to use and affordable TEM microscope that can be used for cryo-EM sample assessment and 3D structure determination [1, 2]. Thermo Fisher Scientific therefore introduced a new TEM microscope dedicated to cryo-EM SPA at the end of last year - the Thermo Scientific ${ }^{\text {TM }}$ Tundra. A new cryo-EM sample loading system was developed to ensure microscope reliability, ease of use, and affordability without the risks of sample deterioration or vacuum failure of the microscope during transfer (a challenge with traditional side entry loading systems).

The new semi-automated system for loading cryo-EM samples (SAL) consists of three main modules as depicted in Figure 1. Semi-automation means that many steps of sample transfer are automated and the only remaining manual action consists in moving the Transfer Device (TD) between a Cryo-loading Workstation (CLW) and the TEM microscope. Users can insert one or two grid boxes with cryo-EM grids into the CLW and put up to 5 selected AutoGrids into special slots for automated picking by the TD. Once the AutoGrids are in the slots, the area is sealed to prevent ice buildup and contamination. All next steps are done in a graphical user interface (GUI) via the built-in touch screen. The GUI indicates readiness of the system for loading an AutoGrid to the TEM. Once confirmed by the user, the TD (vented and flushed with dry nitrogen), is loaded with the selected AutoGrid. Next, TD is pumped to a medium vacuum level. The user is then prompted by the system to transfer the TD to from the CLW to the microscope. The microscope recognizes the inserted TD and automatically starts the loading procedure, which consists of pumping down the airlock and handing over the AutoGrids from the TD gripper to the cryo-stage holder inside the TEM. The TD must then be returned to the CLW to finish the routine. For unloading of the inserted AutoGrid, the TD must be cooled down on the CLW, then transferred mounted onto the TEM again. User initiates removal of the inserted AutoGrid via the touch screen and is guided through the process. Several exchanges of cryo-EM samples can be done before the CLW must be heated and recooled to ensure a contamination free environment for handling the cryo-EM samples.

Features of the new semi-automated loading system:

- Effortless, safe and robust sample exchange: i. Eliminated vacuum failures during grid insertion into TEM; ii. Eliminated sample damage by devitrification; iii. Minimized sample damage by ice contamination

- Reduced complexity: i. Only one manual tweezer step; ii. Only a few minutes to exchange sample; iii. Very little contamination in the microscope - enable $72 \mathrm{hrs}$. data collection

- Connectivity - A used AutoGrid can be retrieved, stored, used later in a higher end TEM (Krios or Glacios)

- Iterative sample screening - Allows a new iterative way of working for instantaneous feedback on biochemistry adjustment

- New UI at on-screen displays navigates the user through the whole process. 


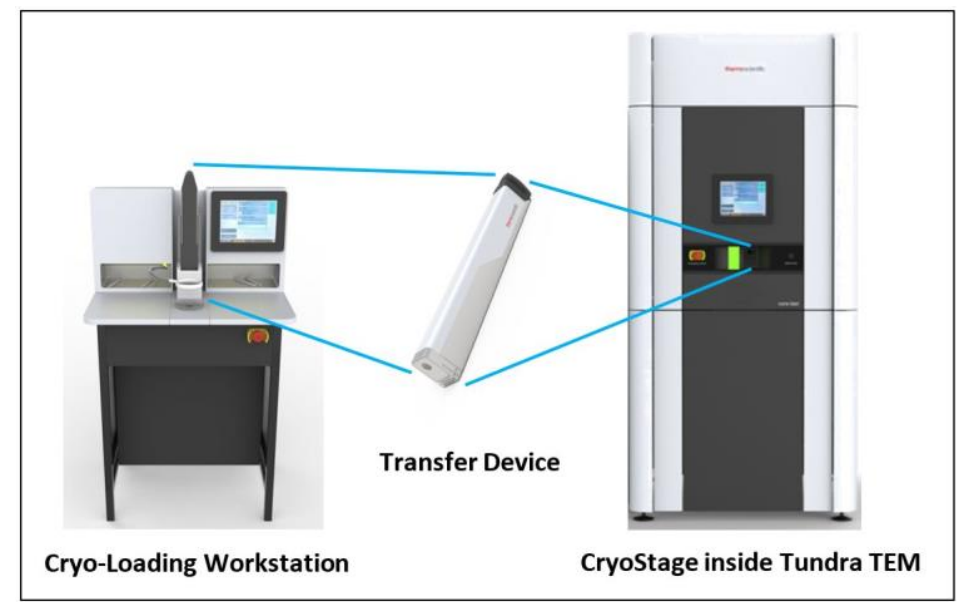

Figure 1. Semi-automated Loader main modules

\section{References}

[1] Peter B. Rosenthal (2019) IUCrJ, 6(6): 988-989.

[2] K. Naydenova et al. (2019) IUCrJ, 6(6): 1086-1098. 